\title{
In situ Transmission Electron Microscopy of Rapidly Solidifying Aluminum
}

\author{
A. Kulovits, ${ }^{*}$ J.M.K. Wiezorek, ${ }^{*}$ T. LaGrange, ${ }^{* *}$ Bryan W. Reed**, and G.H. Campbell** \\ * Department of Mechanical Engineering and Materials Science, University of Pittsburgh, 648 \\ Benedum Hall, 3700 O Hara Street, \\ ** Lawrence Livermore National Laboratory, Physical and Life Science Directorate, Condensed \\ Matter and Materials Division, 7000 East Avenue, P.O. 808 L-356
}

How microstructures evolve during solidification is of scientific and technological importance. Here we studied the morphological and structural changes in metallic thin films induced by rapid liquidsolid transformations with nanoscale spatial and 15 nanosecond temporal resolution using the dynamic transmission electron microscope (DTEM) at Lawrence Livermore National Laboratory (Figure 1a) [1]. This unprecedented time resolution allows the detailed study of phenomena associated with slow as well as ultrafast solidification processes. We discuss the rapid solidification of laser melted zones in nanocrystalline $80 \mathrm{~nm}$ thick Al films deposited on $100 \mathrm{~nm}$ thick amorphous $\mathrm{Si}_{3} \mathrm{~N}_{4}$ membranes. The DTEM experiments were performed by, first, melting the Al films with a single 12ns, 1064nm laser pulse (Figure 1b). After a pre-selected time delay, as short as $15 \mathrm{ns,} \mathrm{an}$ electron pulse then illuminates this previously laser-pulse molten area to acquire image or diffraction data (Figure 1b). Acquiring series of pulsed electron exposures with different time delays permits direct observation of morphological and structural changes during the entire solidification process.

A collage of bright field images taken post mortem shows the morphology of rapidly solidified area with large elongated grains stretching from the edge of the melt pool to its center (Figure 1c). These grains are up to $\sim 10 \mu \mathrm{m}$ long, $\sim 0.5-1.0 \mu \mathrm{m}$ wide and $0.08 \mu \mathrm{m}$ thick, exhibiting very large aspect ratio of $\sim 125: 10: 1$. Figure 2 shows imaging and in diffraction mode snapshots for a series of time delays. Before the melting, the image of the as-deposited nanocrystalline thin film shows minimal contrast due to the low magnification, $\sim 4000 \mathrm{x}$, and $\sim 20 \mathrm{~nm}$ grain size. The corresponding DP shows a ring pattern characteristic of the nanocrystalline $\mathrm{Al}$ film and the diffuse background from the amorphous $\mathrm{Si}_{3} \mathrm{~N}_{4}$ substrate. After $0.5 \mu \mathrm{s}$ time delay no contrast variations are observed in the image and the sharp ring pattern in the DP disappeared, indicating that the Al is in the liquid state. After $5 \mu \mathrm{s}$ the solidification front was in the field of view. Elongated grains formed by rapid lateral solidification and the diffraction spots associated with grains close to a Bragg condition appear in the DP. After solidification the DP showed a reduced diffuse background and distinct diffraction spots instead of diffraction rings, since the number of grains in the field of view was significantly reduced.

Tracing the progress of the solid liquid interface during the transformation using snap shot images we determined that the morphology of the interface is planar, producing morphologically columnar grains, and we estimated the solidification front velocity to be $\sim 3-4 \mathrm{~m} / \mathrm{s}$ (Figure 3). After demonstrating here the feasibility of in-situ TEM study of rapid liquid-solid transformations, future plans include studying effects of interfacial velocity on microstructural morphology by controlled manipulation of the heat extraction and details of stability and morphology of the solid liquid interface in solid solutions. Work was performed under the auspices of the U.S. Department of Energy by the Lawrence Livermore National Laboratory and supported by the Office of Science, Office of Basic Energy Sciences, Division of Materials Sciences and Engineering, of the U.S. Department of Energy under contract No. DE-AC52-07NA27344. 
References

[1] T. LaGrange et al., Appl. Phys. Let. 89 (2006) 0044105

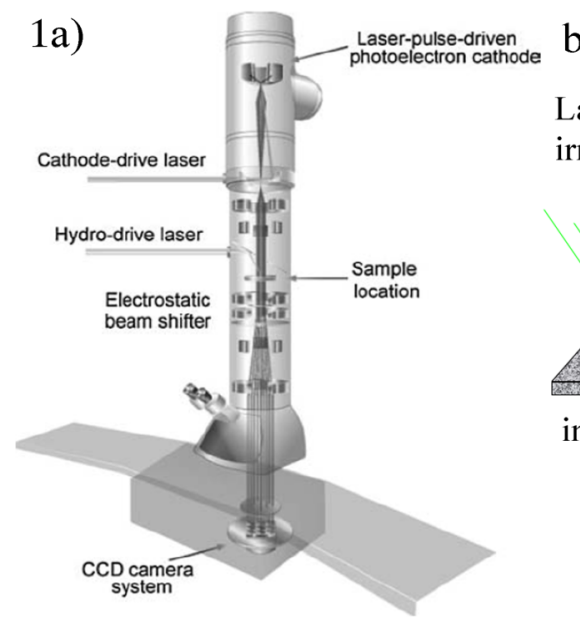

b) c)

Laser pulse irradiation

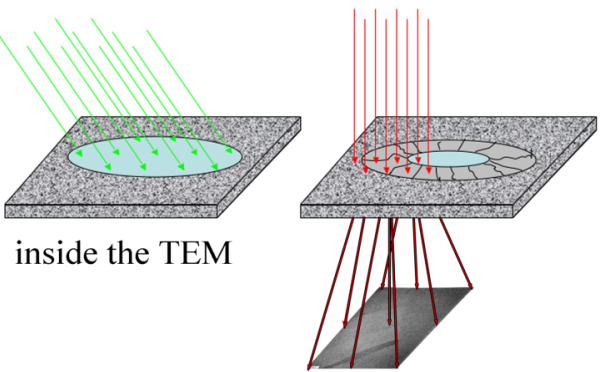

)

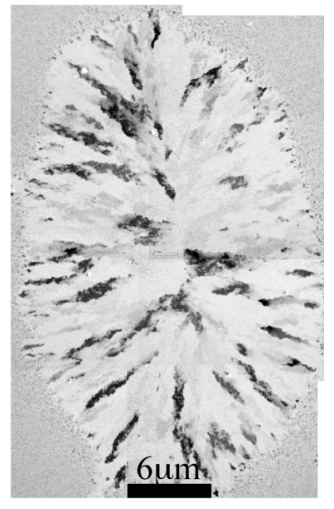

FIG. 1. a) Schematic of the modified TEM instrument, DTEM; b) Schematic of the experimental sequence; c) Bright field image montage of a molten and re-solidified area of Al thin film.
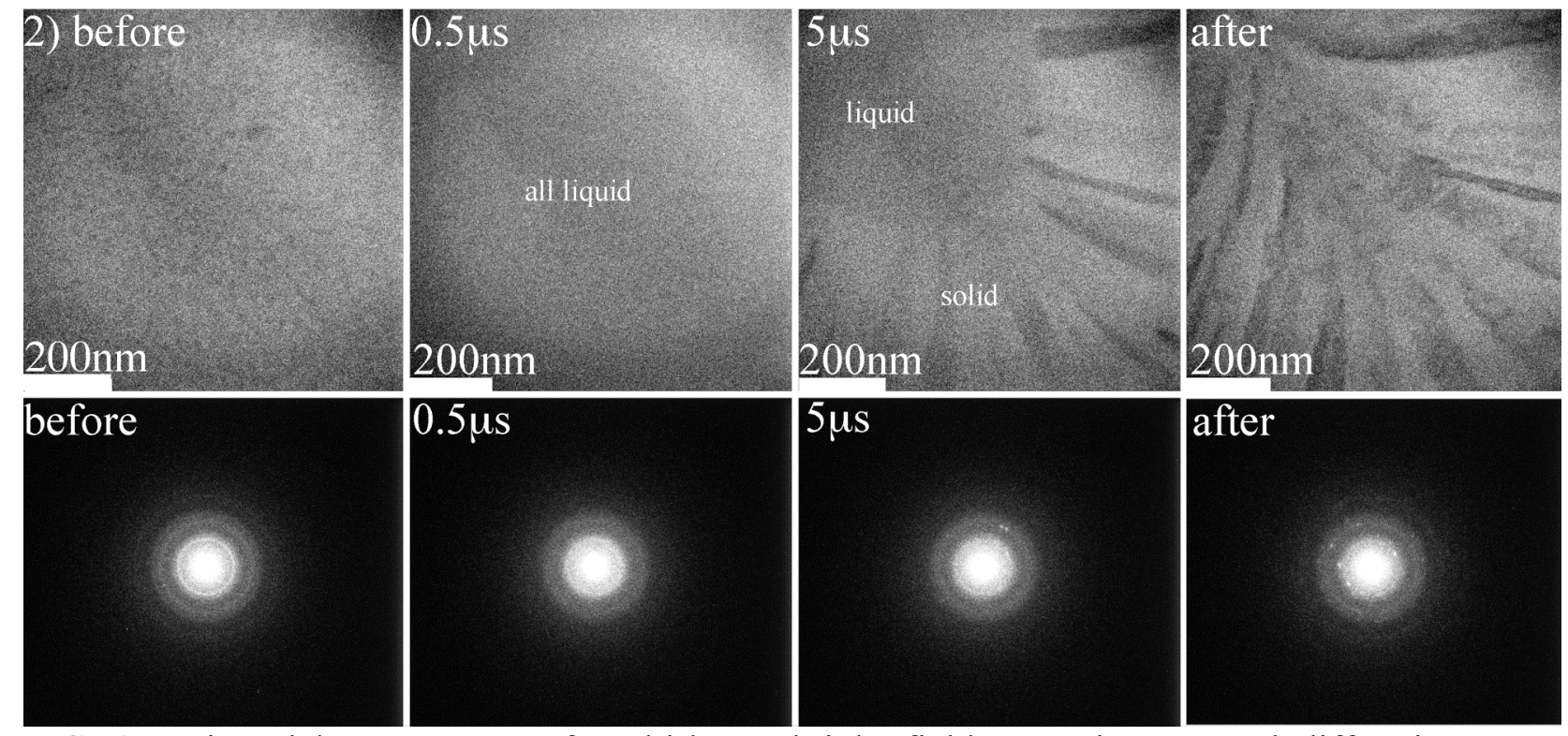

FIG. 2. Time-delay sequence of multi-beam bright field TEM images and diffraction patterns showing morphological and structural evolution during rapid solidification of the Al thin film.

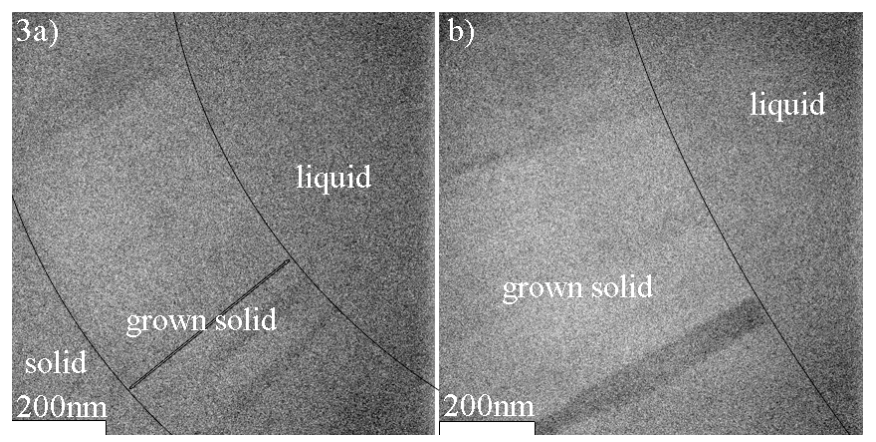

FIG. 3. Images acquired during the rapid solidification process showing the planar nature of the solidification interface moving at speed of $\sim 3.3 \mathrm{~ms}^{-1}$. Curved solid lines approximate the liquid-solid interface. Thick solid line indicates grow direction perpendicular to the solidification front. 